\title{
Direct imaging of orbitals in quantum materials
}

2 Hasan Yavaş, ${ }^{1,2, \dagger}$ Martin Sundermann, ${ }^{1,3}$ Kai Chen,,${ }^{3, \ddagger}$ Andrea Amorese, ${ }^{1,3}$

3 Andrea Severing, ${ }^{1,3}$ Hlynur Gretarsson, ${ }^{1,2}$ Maurits W. Haverkort, ${ }^{4}$ \& Liu Hao Tjeng ${ }^{1, *}$

${ }_{4}{ }^{1}$ Max Planck Institute for Chemical Physics of Solids, Nöthnizer Straße 40, 01187 Dresden, Ger-

many

$6 \quad{ }^{2}$ PETRA III, Deutsches Elektronen-Synchrotron (DESY), Notkestraße 85, 22607 Hamburg, Ger-

many

$8{ }^{3}$ Institute of Physics II, University of Cologne, Zülpicher Straße 77, D-50937 Cologne, Germany

$9{ }^{4}$ Institute for Theoretical Physics, Heidelberg University, Philosophenweg 19, 69120 Heidelberg,

Germany

11 * correspondence should be addressed to L.H.T. (email: hao.tjeng@cpfs.mpg.de)

${ }^{\dagger}$ present address: SLAC National Accelerator Lab., 2575 Sand Hill Rd, Menlo Park, CA 94025,

USA

${ }_{14} \ddagger$ present address: Synchrotron SOLEIL, L’Orme des Merisiers, Saint-Aubin, BP 48, 91192 Gif-

sur-Yvette, France 
The spectacular physical properties of quantum materials based on transition metal, rare earth, and actinide elements continue to challenge our comprehension of solid state physics and chemistry. The electronic states of these materials are dominated by the $d$ and $f$ wave functions intertwined with the strong band formation of the solid. In order to estimate which wave functions contribute to the ground state formation, we have had to rely, until now, on theoretical calculations combined with spectroscopy. Here we show that $s$-core-level nonresonant inelastic $x$-ray scattering ( $s$-NIXS) can directly image the active orbital in real space, without the necessity of any modeling. The power and accuracy of this new technique is shown using the text-book example, $\mathrm{x}^{2}-\mathrm{y}^{2} / 3 z^{2}-\mathbf{r}^{2}$ orbital of the $\mathrm{Ni}^{2+}$ ion in $\mathrm{NiO}$ single crystal. 
The search for new materials with novel properties is commonly focused on materials containing transition metal, rare-earth, and/or actinide elements. The presence of the atomic-like $d$ or $f$ wave functions provide a fruitful playground to generate novel phenomena. ${ }^{1-5}$ The intricate interplay of band formation with the local electron correlation and atomic multiplet effects leads to phases that are nearly isoenergetic, making materials' properties highly tunable by doping, temperature, pressure, or magnetic field. Understanding the behavior of the $d$ and $f$ electrons is essential for designing and controlling novel quantum materials. Therefore, identifying the $d$ or $f$ wave functions that actively participate in the formation of the ground state is crucial. So far, these wave functions have been mostly deduced from optical, x-ray and neutron spectroscopy methods in which spectra must be analyzed and interpreted using theory or modeling. This, however, is also a challenge in and of itself since $a b$-initio calculations hit their limits due to the many-body nature of the problem. Here, we have established an experimental method that circumvents the need for involved analysis, and instead, provides the information as measured. With this technique, we can make a direct image of the active orbital and determine what the atomic-like object looks like in a real solid.

The spectral intensity of the dipole-allowed $s \rightarrow p$ transition depends on the orientation of the electric field polarization vector of the photon relative to the orientation of the $p$ orbital. ${ }^{6}$ Since the $s$ orbital is spherically symmetric, sweeping the polarization vector over all angles yields an angular intensity distribution that directly maps the shape and orientation of the $p$ orbital hole. Yet, material research requires knowledge of $d$ and/or $f$ orbital shapes. As the $s \rightarrow d$ or $s \rightarrow f$ transitions are dipole forbidden, it has been challenging to develop an experimental method that has non vanishing 
matrix elements beyond the dipole limit. However, the relatively new experimental method of nonresonant inelastic x-ray scattering (NIXS), available due to modern synchrotron facilities with high brilliance, has offered new potential.

The interaction of light with matter is given by two terms: a term proportional to the scalar product of the electron momentum operator $\vec{p}$ and the photon vector potential $\vec{A}$, and a term proportional to the vector potential $\vec{A}$ squared. When photon energy matches an atomic resonance, the $\vec{p} \cdot \vec{A}$ term dominates; off-resonance, the interaction is governed by the $\vec{A}^{2}$ term. Focusing on this last term using NIXS, the double differential cross-section $\frac{d^{2} \sigma}{d \Omega d \omega}$ becomes proportional to the dynamical structure factor $\mathrm{S}(\vec{q}, \omega),{ }^{7}$ which contains the material-specific information we are seeking:

$$
S(\vec{q}, \omega)=\sum_{f}\left|\left\langle f\left|e^{i \vec{q} \cdot \vec{r}}\right| i\right\rangle\right|^{2} \delta\left(\hbar \omega_{i}-\hbar \omega_{f}-\hbar \omega\right)
$$

where $|i\rangle$ and $|f\rangle$ denote the (many-body) initial and final states, $\vec{q}=\overrightarrow{k_{i}}-\overrightarrow{k_{f}}$ the transferred momentum, $\hbar \omega=\hbar \omega_{i}-\hbar \omega_{f}$ the transferred energy, and $\vec{k}_{i, f}$ and $\hbar \omega_{i, f}$ the momentum and energy of the incoming and scattered photons, respectively.

Beyond-dipole matrix elements appear in the scattering cross-section when expanding the transition operator $\mathrm{e}^{i \vec{q} \cdot \vec{r}}$ to the $k^{t h}$ order, whereby $k$ denotes the multipole order of the scattering cross-section. $^{7-16}$ The so-called triangular condition and parity rule restrict the number of multipoles to $\left|l_{f}-l_{i}\right| \leq k \leq l_{f}+l_{i}$ and $\left|l_{i}+l_{f}+k\right|=$ even for a $l_{i} \rightarrow l_{f}$ transition (respective orbital momenta of initial and final state). This implies that for a $d \rightarrow f$ transition, only dipole $(k=1)$, octopole $(k=3)$, and triakontadipole $(k=5)$ scattering orders occur, and for $s \rightarrow d$-a dipole-forbidden 
transition- only the quadrupole transition with $k=2$ contributes to $\mathbf{S}(\vec{q}, \omega)$. For small momentum transfers $|\vec{q}|$, the NIXS spectra very much resemble dipole-allowed x-ray absorption spectroscopy (XAS). In other words, the NIXS $2 p / 3 p \rightarrow 3 d$ excitations in transition metal compounds ${ }^{8,10}$ or the $3 d / 4 d \rightarrow 4 f / 5 f$ and $5 d \rightarrow 5 f$ excitations in rare earth and actinide materials ${ }^{9,10}$ exhibit line shapes that are very similar to the ones obtained from XAS. ${ }^{17-21}$ The only difference is that in NIXS, the direction of the momentum transfer $\hat{q}(\vec{q}|| \vec{q} \mid)$ provides the information that is obtained from the electrical vector polarization in XAS. However, for large $|\vec{q}|$, for the same metal ion, the $p \rightarrow d$ or $d \rightarrow f$ transitions yield a different spectral distribution with additional features that cannot be seen in a dipole-based XAS experiment. ${ }^{7-16,22,23}$ Moreover, the dipole-forbidden $s \rightarrow d$ or $s \rightarrow f$ transitions (quadrupolar or octopolar, respectively) now have non-vanishing matrix elements, and consequently are allowed and become visible. 


\section{Results}

The novelty of our approach is to exploit these $s$-core-level transitions involving our search for a new method to determine - quantitatively and model free - the local valence orbitals that make up the electronic structure of $d$ and $f$ containing quantum materials. We investigated the $s \rightarrow d$ transition in an inelastic x-ray scattering experiment (s-NIXS) at large momentum transfers $|\vec{q}|$ and map the quadrupolar scattering intensity as a function of the direction of the momentum transfer $\hat{q}$ relative to the crystal lattice. We used a single crystal of $\mathrm{NiO}$, an antiferromagnetic insulator, ${ }^{24}$ with a $\mathrm{Ni} d^{8}$ configuration as a model system; and the large momentum transfers were guaranteed by high scattering angles and hard x-rays.

In our experimental setup as illustrated in $\mathrm{Fig} \cdot 1, \mathrm{~S}(\vec{q}, \omega)$ of the $\mathrm{NiO}$ sample was recorded as a function of the sample angle $\varphi$, here defined as the angle between the fixed momentum transfer vector $\vec{q}$ and the NiO surface normal (see Methods). Fig. 2 shows a compilation of NIXS spectra measured for many different sample angles. The spectra show the $\mathbf{M}_{2,3}$ edge $(3 p \rightarrow 3 d)$ of nickel at around $70 \mathrm{eV}$ and, most importantly, the dipole-forbidden $\mathbf{M}_{1}(3 s \rightarrow 3 d)$ excitations at around $110 \mathrm{eV}$, overlaid on the broad Compton profile. The signal to background ratio is excellent in the energy range of the $\mathrm{M}_{1}$ edge. A close-up of this edge and its directional dependence on $\vec{q}$ along $\vec{q} \|[001]$ and $\vec{q} \|[100]$ are displayed in Fig. $3 \mathrm{a}$, and for $\vec{q}$ along $\vec{q} \|[001]$ and $\vec{q} \|[110]$ in Fig. $3 \mathrm{~b}$ and c. In the close-up plots, the Compton profile has been subtracted using a simple linear background (see Methods).

To quantitatively analyze the $3 s \rightarrow 3 d$ transition's directional dependence, we determined 
the integrated intensity of each spectrum in Fig. 3 and placed it on a polar plot as displayed in (Fig.4). Panel(a) shows the data points for $\vec{q}$ sweeping in the [001]-[100] plane (orange), and panel(b) for $\vec{q}$ in the [001]-[110] plane (green). They fall accurately on top of the orbital shapes which denote 'cuts' through the [001]-[100] (orange) and [001]-[110] (green) planes of the calculated three-dimensional orbital hole density (square of the wave function) of the Ni highspin $3 d^{8}$ configuration in octahedral coordination, namely the ${ }^{3} A_{2} 3 d\left(x^{2}-y^{2}\right) 3 d\left(3 z^{2}-r^{2}\right)$ as shown in Fig. 4 (c). This means that, for the first time, we have generated a purely experimental method that can directly visualize the fundamental atomic-like quantum mechanical objects in solids. The information that we have obtained is extremely detailed; for example, we can clearly see the small lobes of the $3 d\left(3 z^{2}-r^{2}\right)$ contribution in Fig. $4(\mathrm{~b})$. We would like to remark that the $3 d\left(x^{2}-\right.$ $\left.y^{2}\right)$ contribution vanishes in the the [001]-[110] plane, Fig. $4(\mathrm{~b})$, while both the $3 d\left(x^{2}-y^{2}\right)$ and $3 d\left(3 z^{2}-r^{2}\right)$ contribute in the [001]-[100] plane, Fig. 4 (a). Here we note that we have used two orbital shapes in Fig. 4(b): the blue dashed line is the $3 d\left(x^{2}-y^{2}\right) 3 d\left(3 z^{2}-r^{2}\right)$ function and the solid line is the same function convoluted with the angular acceptance of the $3 \times 4$ analyzers we used in our experiment (see Methods). The near perfect agreement further demonstrates the accuracy of the method.

\section{Discussion}

The directional dependence of the integrated $s$-NIXS intensity at the Ni $M_{1}$ edge $(3 s \rightarrow 3 d)$ directly maps the local orbital hole density of the ion in the ground state. There is no need to carry out multiplet analysis of the spectral line shape to extract this information, in contrast to, for example, the non- $s$ edges (e.g. $L_{2,3}(2 p), M_{2,3}(3 p), M_{4,5}(3 d), N_{4,5}(4 d)$, and $\left.O_{4,5}(5 d)\right)$ in both NIXS ${ }^{7-13,16,22,23}$ 
and XAS experiments ${ }^{17-21}$ as well as in core-level photoemission. ${ }^{25}$ The reason is fundamental: The $M_{1}(3 s \rightarrow 3 d)$ quadrupolar excitation process involves a spherically symmetric $s$ orbital, so the angular distribution of intensity is solely determined by the hole charge distribution in the initial state with respect to the momentum transfer $\vec{q}$. This is similar to the dipole-allowed $s \rightarrow p$ transition in XAS, where an angular sweep of the polarization dependence maps out the orientation of the $p$ hole directly. We would like to emphasize that details of the $s$-NIXS final states do not matter because the information is extracted from the integrated intensity of the spectra (i.e. from the sum of the intensities of all final states). As a result, only the properties of the initial state are probed. This is true for both localized and itinerant systems. The procedure is analogous to using spectral sum rules to extract expectation values of the relevant quantum numbers of the system in the ground state. ${ }^{20,26-28}$ The power of $s$-NIXS, as compared to XAS, is that it allows transitions not only from $s$-to- $p$, but also from $s$-to- $d$ and $s$-to- $f$ due to the possibility of going beyond the dipole limit when using large momentum transfers $|\vec{q}|$.

The $s$-NIXS process involves a core hole, meaning that both the electronic structure of the system and consequently the measured valence hole are projected locally. The intensity distribution is not what would be measured in an x-ray diffraction (XRD) experiment, even if such an experiment could be carried out with sufficient accuracy. In fact, it would be extremely difficult for transition metal, rare earth, and actinide compounds to be measured with the desired accuracy in XRD due to their relatively small number of valence electrons with respect to core electrons. $s$ NIXS provides information complementary to that from an XRD experiment by elucidating which local orbital or atomic wave function is active. 
The $s$-NIXS method presented here is not limited to ionic materials. In cases where configuration interaction effects play an important role due to covalency or itineracy, the image of the probed local orbital will reflect these effects directly. For example, in octahedral coordination one may detect $e_{g}$ and $t_{2 g}$ orbital occupation ratios which are quite different from the ones based on the formal valency. The strength of $s$-core-level NIXS is that the information is extracted from the $\overrightarrow{q-}$ directional dependence of the integrated intensity and not from the line shape of the spectra. Thus, the details of the final states are no longer important, rendering complex configuration interaction calculations unnecessary. The sole $\vec{q}$-directional dependence is rooted in the spherical symmetry of the $s$-core hole.

To conclude, we have directly imaged one of the fundamental quantum mechanical objects in crystals, namely, a $d$-orbital, which is derived purely mathematically from first principles. We have revealed that non-resonant inelastic x-ray scattering involving an $s$-core level is an extremely powerful and accurate experimental method to determine the local orbital in the ground state. Albeit low cross-section, the excellent signal to background ratio allows for highly reliable results. The procedure relies on the integrated intensity of the signal, which tremendously simplifies the interpretation as there is no need to carry out multiplet analysis of the spectral line shape to extract the desired information. The method is element specific, which is invaluable for unraveling the different origins for electron correlation effects in complex materials. Since the probing photons have high penetrating power, the measurements are bulk sensitive, and can be performed with complex sample environments (e.g. small samples, high pressures, high/low temperatures). A clear example of an immediately relevant application of $s$-NIXS in the field of correlated electron 
physics would be to identify the entangled spin-orbit states in ruthenium and iridium materials. ${ }^{29,30}$

We also believe that this method opens up new opportunities for the study of a wide range of $d$ and $f$ electron containing materials, where knowledge of their local wave function is of central importance to reveal their underlying physics and thus provide guidance for the design of new quantum materials.

\section{Materials and Methods}

Experiment: Non-resonant inelastic x-ray scattering (NIXS) measurements were performed at the High-Resolution Dynamics Beamline P01 of PETRA-III synchrotron in Hamburg, Germany. Fig. 1 illustrates the experimental setup, showing the incoming beam $\left(\vec{k}_{i}, \omega_{i}\right)$, sample, scattered beam $\left(\vec{k}_{f}, \omega_{f}\right)$, and the corresponding momentum transfer vector $(\vec{q})$. The energy of the x-ray photon beam incident on the sample was tuned with a $\mathrm{Si}(311)$ double-reflection crystal monochromator (DCM). The photons scattered from the sample were collected and energy-analyzed by an array of twelve spherically bent $\operatorname{Si}(660)$ crystal analyzers. The analyzers are arranged in a $3 \times 4$ configuration. The energy of the analyzers $\left(\hbar \omega_{f}\right)$ was fixed at $9690 \mathrm{eV}$; the energy loss spectra were measured by scanning the energy of the DCM $\left(\hbar \omega_{i}\right)$. Each analyzer signal was individually recorded by a position-sensitive custom-made LAMBDA detector. The energy calibration was regularly checked by measuring the zero-energy-loss position of each spectrum. The best possible energy resolution was guaranteed by pixel-wise analysis of the detector recordings and measured as $0.7 \mathrm{eV}$ (FWHM).

The positioning of the analyzer array determines the momentum transfer vector and the cor- 
responding scattering triangle, which is defined by the incident and scattered photon momentum vectors, $\vec{k}_{i}$ and $\vec{k}_{f}$, respectively. The large scattering angle $\left(2 \theta \approx 155^{\circ}\right)$ chosen for the current study assured a large momentum transfer of $|\vec{q}|=(9.6 \pm 0.1) \AA^{-1}$ when averaged over all analyzers. $\vec{k}_{f}$ and $2 \theta$ were kept constant by fixing the energy and the position of the analyzer array. Since the energy transfer range of interest (100 to $120 \mathrm{eV}$ ) was small with respect to the incident and final energies $(\sim 9700 \mathrm{eV})$, variation of $\vec{k}_{i}$ during energy scanning was insignificant. This guaranteed that the scattering triangle was virtually unchanged throughout the course of the experiment with $|\vec{q}| \approx$ constant.

Sample: $\mathrm{NiO}$ single crystal (SurfaceNet, Germany) was kept at $\mathrm{T}=20 \mathrm{~K}$ throughout the experiment. It was aligned as in $\mathrm{Fig} .1$ and rotated by angle $\varphi$ around an axis perpendicular the [010] lattice direction; $\vec{q} \|[001](\varphi=0)$ corresponds to specular geometry. Energy scans were taken for many values of $\varphi$ so that the directional dependence of $S(\vec{q}, \omega)$ could be measured for $\vec{q}$ sweeping between $\vec{q} \|[001]$ and $\vec{q} \|[100]$. For the second set of measurements, the crystal was reoriented to evaluate $S(\vec{q}, \omega)$ on the $\vec{q}\|[001]-\vec{q}\|[110]$. This time, the axis of rotation was along [1]10]. Each $\varphi$ data point takes about 90 minutes in which the elastic peak, Compton profile, and the $s$-NIXS edge were recorded. The data collection for the NiO project took about three days.

Data Treatment: The data were normalized to the Compton peak at about $350 \mathrm{eV}$ energy transfer (see Fig. 2). Subsequently, a linear background was subtracted from each spectrum in order to account for the Compton scattering in the energy range of the $\mathrm{Ni} \mathrm{M}_{1}$ edge.

We would like to note that the line shape of the Compton profile does not change with angle 
as can been from Fig. 2. This is fully consistent with the fact that the scattering geometry is kept constant while rotating the sample. What does vary is the intensity of the Compton. This is related to how the x-rays are absorbed when entering the sample and when scattered out of the sample. This process can be modeled quantitatively since the NiO crystals used have well defined (flat and shiny) surfaces. The result of this modeling and the comparison with the experiment is displayed in Fig. 5. The calculations were done for the actual scattering geometry of $2 \theta=155^{\circ}$ so that for $\varphi=77.5^{\circ}$ specular geometry is fulfilled. Turning the sample towards (away from) the analyzer, increases (decreases) the intensity. One can observe a very good overall match between the experiment and the modeling. Slight deviations of the order of a few percent can be ascribed to imperfections in the kapton-glue-aluminium windows (i.e. thickness variations) of our cryostat. Figure 5 therefore demonstrates that we understand fully the details of the scattering process, and in particular, that the intensity variations of the Compton profile is due to the absorption processes in the sample (and also in the cryostat windows). We therefore can safely use the Compton profile to normalize our $s$-NIXS spectra.

Since the analyzer array $(3 \times 4)$ is spread over a finite solid angle, each measured spectrum $\mathrm{S}(\vec{q}, \omega)$ includes an array of momentum transfer vectors $\vec{q}$ corresponding to individual analyzers. In this case, taking an average $\vec{q}$ does not work for directions where the orbital wave function varies significantly for small angular changes (i.e. small lobes of the $\left.3 d\left(3 z^{2}-r^{2}\right)\right)$. The theoretical orbital wave function should be convoluted with the angular spread of the analyzer array to reflect this effect. The inlet on Fig. 4(b) demonstrates the theoretical function (blue dashed line) and the convoluted function (solid line), which agrees well with the data points. 


\section{Data availability:}

The data that support the findings of this study are available from the corresponding authors upon request.

1. Cava, R. J. Oxide superconductors. Journal of the American Ceramic Society 83, 5-28 (2008).

2. Khomskii, D. I. Transition Metal Compounds (Cambridge University Press, 2014).

3. Keimer, B., Kivelson, S. A., Norman, M. R., Uchida, S. \& Zaanen, J. From quantum matter to high-temperature superconductivity in copper oxides. Nature 518, 179 (2015).

4. Wirth, S. \& Steglich, F. Exploring heavy fermions from macroscopic to microscopic length scales. Nature Reviews Materials 1, 16066 (2016).

5. Pfleiderer, C. Superconducting phases of $f$-electron compounds. Rev. Mod. Phys. 81, 15511624 (2009).

6. Fowles, G. R. Introduction to Modern Optics (Holt, Rinehart and Wisnton, Inc., 1968).

7. Schülke, W. Electron dynamics by Inelastic x-ray scattering (Oxford University Press, 2008).

8. Haverkort, M. W., Tanaka, A., Tjeng, L. H. \& Sawatzky, G. A. Nonresonant inelastic x-ray scattering involving excitonic excitations: The examples of $\mathrm{NiO}$ and $\mathrm{CoO}$. Phys. Rev. Lett. 99, $257401(2007)$.

9. Gordon, R. A. et al. High multipole transitions in NIXS: Valence and hybridization in $4 \mathrm{f}$ systems. EPL (Europhysics Letters) 81, 26004 (2008). 
10. Gordon, R. A., Haverkort, M. W., SenGupta, S. \& Sawatzky, G. A. Orientation-dependent X-ray Raman scattering from cubic crystals: Natural linear dichroism in $\mathrm{MnO}$ and $\mathrm{CeO}_{2} . J$. Phys. Conf. Ser. 190, 012047 (2009).

11. Bradley, J. A. et al. Probing electronic correlations in actinide materials using multipolar transitions. Phys. Rev. B 81, 193104 (2010).

12. Caciuffo, R. et al. Uranium $5 d-5 f$ electric-multipole transitions probed by nonresonant inelastic x-ray scattering. Phys. Rev. B 81, 195104 (2010).

13. Bradley, J. A., Moore, K. T., van der Laan, G., Bradley, J. P. \& Gordon, R. A. Core and shallow-core $d$ - to $f$-shell excitations in rare-earth metals. Phys. Rev. B 84, 205105 (2011).

14. van der Laan, G. Spin-orbit sum rule for electric multipole transitions in nonresonant inelastic X-ray scattering. Phys. Rev. Lett. 108, 077401 (2012).

15. van der Laan, G. Nonresonant inelastic x-ray scattering from actinides and rare earths. Phys. Rev. B 86, 035138 (2012).

16. Willers, T. et al. Determining the in-plane orientation of the ground-state orbital of $\mathrm{CeCu}_{2} \mathrm{Si}_{2}$. Phys. Rev. Lett. 109, 046401 (2012).

17. Chen, C. T. et al. Out-of-plane orbital characters of intrinsic and doped holes in $\mathrm{La}_{2-x} \mathrm{Sr}_{x} \mathrm{CuO}_{4}$. Phys. Rev. Lett. 68, 2543-2546 (1992).

18. de Groot, F. X-ray absorption and dichroism of transition metals and their compounds. J. Electron Spectros. 67, 529 - 622 (1994). 
19. Tanaka, A. \& Jo, T. Resonant $3 d, 3 p$ and $3 s$ photoemission in transition metal oxides predicted at $2 p$ threshold. J. Phys. Soc. Jpn. 63, 2788-2807 (1994).

20. Csiszar, S. I. et al. Controlling orbital moment and spin orientation in CoO layers by strain. Phys. Rev. Lett. 95, 187205 (2005).

21. Hansmann, P. et al. Determining the crystal-field ground state in rare earth heavy fermion materials using soft-x-ray absorption spectroscopy. Phys. Rev. Lett. 100, 066405 (2008).

22. Sundermann, M. et al. Direct bulk-sensitive probe of $5 f$ symmetry in $\mathrm{URu}_{2} \mathrm{Si}_{2}$. Proc. Nat. Acad. Science. U.S.A. 113, 13989 (2016).

23. Sundermann, M. et al. $4 f$ crystal field ground state of the strongly correlated topological insulator $\mathrm{SmB}_{6}$. Phys. Rev. Lett. 120, 016402 (2018).

24. Sawatzky, G. A. \& Allen, J. W. Magnitude and origin of the band gap in NiO. Phys. Rev. Lett. 53, 2339-2342 (1984).

25. Hamamoto, S. et al. Linear dichroism in angle-resolved core-level photoemission spectra reflecting $4 \mathrm{f}$ ground-state symmetry of strongly correlated cubic Pr compounds. J. Phys. Soc. Jpn. 86, 123703 (2017).

26. Thole, B. T., Carra, P., Sette, F. \& van der Laan, G. X-ray circular dichroism as a probe of orbital magnetization. Phys. Rev. Lett. 68, 1943-1946 (1992).

27. Carra, P., Thole, B. T., Altarelli, M. \& Wang, X. X-ray circular dichroism and local magnetic fields. Phys. Rev. Lett. 70, 694-697 (1993). 
28. Chen, C. T. et al. Experimental confirmation of the x-ray magnetic circular dichroism sum rules for iron and cobalt. Phys. Rev. Lett. 75, 152-155 (1995).

29. Jackeli, G. \& Khaliullin, G. Mott insulators in the strong spin-orbit coupling limit: From heisenberg to a quantum compass and kitaev models. Phys. Rev. Lett. 102, 017205 (2009).

30. Jain, A. et al. Higgs mode and its decay in a two-dimensional antiferromagnet. Nat. Phys. 13, 633 (2017).

Acknowledgements M.S., K.C., and A.S. gratefully acknowledge support from the German funding agency DFG under Grant No SE1441-4-1.

Contributions L.H.T. and M.W.H. initiated the project, H.Y., M.S., K.C., A.A. and H.G. performed the experiment and analyzed the data, H.Y., A.S., M.W.H. and L.H.T. wrote the manuscript with input from all authors.

Competing Interests The authors declare that they have no competing financial interests. 
Fig. 1. Scattering geometry. The scattering geometry is defined by the incoming and the scattered beam, $k_{i}, \omega_{i}$ and $k_{f}, \omega_{f}$, respectively (dashed green arrows). This geometry, which outlines the scattering triangle, remains fixed throughout the measurements. The single crystal sample (blue) is rotated around an axis perpendicular to the scattering plane (maroon) by an angle $\varphi$, and for each $\varphi$ an inelastic spectrum is collected. Here, $\varphi=0$ refers to $\vec{q} \|[001]$ (specular geometry).

Fig. 2. Experimental NIXS spectra of NiO. Compilation of spectra, which were collected for a variety of crystal rotations $\varphi$ with respect to the geometrically fixed momentum transfer vector $\vec{q}$. The graph shows all the spectra. The Compton profile peaks at approximately $350 \mathrm{eV}$ and is used for data normalization. The dipole-allowed $\mathrm{Ni} \mathrm{M}_{2,3}(3 p \rightarrow 3 d)$ edge at around $70 \mathrm{eV}$ energy transfer and the dipole-forbidden $\mathrm{Ni} \mathrm{M}_{1}(3 s \rightarrow 3 d)$ excitations at around $110 \mathrm{eV}$ can be clearly observed. The data exhibit an excellent signal to background (Compton) ratio in the energy range of the $\mathrm{Ni}$ edges, rendering NIXS as a high-contrast experiment.

Fig. 3. Ni $M_{1}(3 s \rightarrow 3 d)$ edge spectra. A close-up view of the relevant range of the spectra after background subtraction and normalization. Panel (a) shows data for momentum transfer vector $\vec{q}$ sweeping on a plane defined by $\vec{q} \|[001]$ and $\vec{q} \|[100]$ and (b-c) on a plane defined by $\vec{q} \|[001]$ and $\vec{q} \|[110]$. Spectra corresponding to major axes are marked accordingly, and the spectra in between are plotted sequentially. Panel (b) shows the spectra, where $\vec{q}$ is sweeping clockwise from $\vec{q} \|[00 \overline{1}]$, passing $\vec{q} \|[\overline{1} \overline{1} 0]$, and ending where the projection of the orbital function vanishes. Similarly, panel (c) shows data, where $\vec{q}$ is sweeping counterclockwise from $\vec{q} \|[00 \overline{1}]$ towards $\vec{q} \|[001]$. The data are vertically shifted for clarity. The scattering intensity of the $\mathrm{M}_{1}$ edge excitations ( $3 s \rightarrow 3 d$ ) depends 
strongly on the relative orientation of momentum transfer and crystallographic direction in the $\mathrm{NiO}$ single crystal.

Fig. 4. Orbital shape of ${ }^{3} A_{2} 3 d\left(x^{2}-y^{2}\right) 3 d\left(3 z^{2}-r^{2}\right)$ hole density. Three-dimensional hole density distribution of the Ni high-spin $3 d^{8}$ configuration (c). Projection of the three-dimensional orbital shape on two planes defined by (a) [001] and [100] and (b) [001] and [110]. Data points on polar plots (a-b) are integrated intensities for $\mathrm{Ni} M_{1}(3 s \rightarrow 3 d)$ for corresponding $\varphi$, which is the angle between the momentum transfer vector $\vec{q}$ and the surface normal vector [001] for both cases (a-b). For (a) the sample is rotated such that the $\vec{q}$ sweeps between [001] and [100], and for (b) $\vec{q}$ sweeps between [001] and [110]. Inset in (b) demonstrates the theoretical orbital function (blue dashed line) and the corrected function as a result of an angular convolution with the $3 \times 4$ analyzer array. For (a), the correction was insignificant.

Fig. 5. Calculated and measured Compton intensity as function of sample angle $\varphi$ with respect to specular geometry $\left(\varphi=77.5^{\circ}\right)$ for a scattering angle $2 \theta=155^{\circ}$. 